\title{
High-dose folic acid improves endothelial function by increasing tetrahydrobiopterin and decreasing homocysteine levels
}

\author{
MIN ZHANG ${ }^{*}$, JINLIN WEN, XIANGJIANG WANG ${ }^{*}$ and CHUN XIAO \\ Department of Cardiovascular Medicine, The Third People's Hospital, Guangzhou Medical University, \\ Huizhou, Guangdong 516002, P.R. China
}

Received October 9, 2013; Accepted April 8, 2014

DOI: $10.3892 / \mathrm{mmr} .2014 .2332$

\begin{abstract}
The aim of this study was to investigate the effect of folic acid (FA) on tetrahydrobiopterin (BH4), neopterin, nitric oxide (NO) and homocysteine (Hcy) levels in endothelial cells. Human umbilical vein endothelial cells (HUVECs) were cultured in vitro in the presence or absence of Hcy. The effect of various doses of FA on Hcy, BH4, neopterin and NO concentrations in HUVECs was then assessed. In the 5 and $10 \mathrm{nmol} / \mathrm{l} \mathrm{FA}$ treatment groups, FA was found to significantly increase the levels of BH4 $(10.56 \pm 3.86$ and $11.23 \pm 2.1919 \mathrm{pmol} / \mathrm{g}$ vs $6.32+2.87 \mathrm{nmol} / \mathrm{g} ; \mathrm{P}<0.05$ vs. control) and NO production $(37.86 \pm 12.34 \mathrm{nmol} / 1,38.45 \pm 11.23 \mathrm{nmol} / 1 \mathrm{vs}$ $26.21 \pm 9.24 \mathrm{nmol} / \mathrm{l} ; \mathrm{P}<0.001$ vs. paired Hcy group), but reduce the levels of Hcy $(132.87 \pm 29.67$ and $140.87 \pm 26.76 \mathrm{nmol} / \mathrm{l}$ vs. $165.23 \pm 30.56 \mathrm{nmol} / \mathrm{l} ; \mathrm{P}<0.05$ vs. Hcy group). No significant differences were observed in neopterin levels among the different groups of HUVECs. In conclusion, high doses of FA may be capable of protecting endothelial cells through reducing levels of Hcy and increasing $\mathrm{BH} 4$ and $\mathrm{NO}$ production.
\end{abstract}

\section{Introduction}

Nitric oxide (NO) is generated by endothelial nitric oxide synthase (eNOS) and has diverse biological functions, including anti-inflammatory effects, inhibition of platelet accumulation and hypertension, anti-atherosclerosis effects and attenuation of diabetes and insulin resistance. Therefore, NO is critical for maintaining cardiovascular homeostasis. Endothelial NO release is affected by various factors, such as

Correspondence to: Mr. Chun Xiao, Department of Cardiovascular Medicine, The Third People's Hospital, Guangzhou Medical University, 1 Xuebei Avenue, Huizhou, Guangdong 516002, P.R. China

E-mail: zhangminwin521@163.com

${ }^{*}$ Contributed equally

Key words: folate, homocysteine, nitric oxide, tetrahydrobiopterin, neopterin hormones, the inflammatory state, cytokines and mechanical stress. In addition to the regulation of eNOS at a substrate level, through competition between L-arginine and the competitive inhibitor endogenous asymmetric dimethylarginine, the activity and function of eNOS is regulated by its cofactor, tetrahydrobiopterin (BH4). Biopterin has two isoforms, $\mathrm{BH} 4$ and its enzyme-recycling oxidized form, 7,8-dihydrobiopterin (BH2). Upon BH4 deficiency or the addition of $\mathrm{BH} 2$, endothelial cells metabolize L-arginine to produce reactive oxygen species (ROS) through eNOS uncoupling. BH4 supplementation recovers eNOS activity and increases NO production. Thus, the concentration of $\mathrm{BH} 4$ and the relative concentrations of $\mathrm{BH} 4$ and $\mathrm{BH} 2$ regulate eNOS bioactivity and endothelial function.

$\mathrm{BH} 4$ is derived from synthesis and redox recycling in vivo. The former occurs through a biosynthetic pathway involving guanosine triphosphate cyclohydrolase I, which catalyzes the formation of dihydroneopterin triphosphate from guanosine triphosphate (GTP), and two further steps catalyzed by 6-pyruvoyltetrahydropterin synthase and sepiapterin reductase. In addition, $\mathrm{BH} 2$, a by-product of the synthesis pathway, can be converted to BH4 by dihydrofolate reductase (DHFR). The generation of $\mathrm{BH} 4$ through redox recycling is catalyzed by dihydropteridine reductase (DHPR). Therefore, the generation of BH4 through synthesis and redox recycling involves the reductases DHFR and DHPR, respectively, whose activities are dependent on 5-methyltetrahydrofolate (1). Folate enhances the binding of BH4 to NOS through a pteridine-binding domain and then 5-methyltetrahydrofolate facilitates the electron transfer by $\mathrm{BH} 4$ from the NOS reductase domain to the heme. Therefore, folic acid (FA) enhances the chemical synthesis and stabilization of BH4 (2).

Hyperhomocysteine damages endothelial cells in the vasculature through increased formation of ROS and inflammation, thereby increasing the likelihood of cardiac diseases and stroke. Hyperhomocysteinemia also promotes low-density lipoprotein (LDL) oxidation and internalization by macrophages, which is the initial step in atherosclerosis (3-5). Folate has been identified to reduce the level of homocysteine (Hcy), but the clinical application of folate is controversial, with certain studies demonstrating that folate does not affect the process of atherosclerosis directly (6-7). In the present study, the protective effect of FA on endothelial cells was investigated. 


\section{Materials and methods}

Chemicals. Hcy, BH4 and neopterin standard substances were purchased from Sigma-Aldrich (St. Louis, MO, USA), as were the cell culture media. All other chemicals were obtained from the Tianjin Chemical Raw Materials Company (Tianjin, China). Human umbilical vein endothelial cells (HUVECs) were purchased from American Type Culture Collection (Manassas, VA, USA).

Determination of total Hcy ( $\mathrm{tHcy}$ ). tHcy levels were measured using a fluorescence polarization immunoassay adapted to the IMx ${ }^{\circledast}$ analyzer (Abbott Diagnostics, Abbott Park, IL, USA).

Measurement of biopterins and neopterin. Measurement of $\mathrm{BH} 4$ and $\mathrm{BH} 2$ was performed using high-performance liquid chromatography (HPLC) analysis, following iodine oxidation in acidic or alkaline conditions, as previously described (8). Briefly, cell pellets were lysed in cold extract buffer [50 mM Tris- $\mathrm{HCl}$ (pH 7.4), $1 \mathrm{mM}$ dithiothreitol and $1 \mathrm{mM}$ EDTA, containing $0.1 \mathrm{mM}$ neopterin as an internal recovery standard]. Protein concentration was measured using the Bio-Rad protein assay (Bio-Rad, Hercules, CA, USA). Proteins were extracted with the addition of $10 \mathrm{ml} 1: 1$ mixture of $1.5 \mathrm{M}$ $\mathrm{HClO}_{4}$ and $2 \mathrm{M} \mathrm{H}_{3} \mathrm{PO}_{4}$ to $90-\mu \mathrm{l}$ extracts, followed by centrifugation. Subsequent to deproteination, paired samples were subjected to oxidation with $1 \%$ iodine $/ 2 \%$ potassium iodide under acidic and basic conditions. Biopterin concentrations were determined using HPLC in 5\% methanol/95\% water with a Spherisorb ${ }^{\circledR}$ ODS-1 column (Waters Ltd., Elstree, $\mathrm{UK})$ and fluorescence detection $(350 \mathrm{~nm}$ excitation and $450 \mathrm{~nm}$ emission). For each assay, a standard curve of fresh BH4 (range, $10^{-7}-10^{-9} \mathrm{M}$ ) was included using the same extract buffer. $\mathrm{BH} 4$ concentration was calculated by subtracting $\mathrm{BH} 2$ plus oxidized biopterin from the total biopterins. Neopterin concentration was measured in the same manner, using a neopterin standard curve.

Determination of NO production. NO is rapidly converted to nitrite $\left(\mathrm{NO}_{2}^{-}\right)$and nitrate $\left(\mathrm{NO}_{3}^{-}\right)$; therefore, the total concentration of $\mathrm{NO}_{2}{ }^{-}$and $\mathrm{NO}_{3}{ }^{-}$is used as a quantitative measure of NO production. An NO assay kit was purchased from Biyuntian Biotech Co., Ltd. (Shanghai, China) and NO production was calculated in accordance with the manufacturer's instructions.

Cell culture. Human umbilical vein endothelial cells (HUVECs) were cultured in M199 media containing 10\% fetal bovine serum (FBS) in $75-\mathrm{cm}^{2}$ flasks, and were maintained in an incubator at $37^{\circ} \mathrm{C}$ with $5 \% \mathrm{CO}_{2}$. Upon reaching 90\% confluence, HUVECs were passaged using $0.25 \%$ trypsin and were synchronized. In brief, following growth to $70-80 \%$ confluence, HUVECs were placed in reduced serum (0.5\% FBS) media for $12 \mathrm{~h}$ and, subsequent to attachment, the media were changed to media without serum. Following synchronization, HUVECs were randomly divided into normal (not stimulated by Hcy) and Hcy-stimulated groups, and treated with various concentrations of FA $(0.1,1,5$ and $10 \mathrm{~mol} / \mathrm{l})$. HUVECs were then lysed on ice and the culture medium, including the lysate, was collected. HPLC was performed in order to measure the effect of exogenous FA on HUVEC activity.

Statistical analysis. Experiments were performed at least in triplicate. Statistical analyses were performed using the SPSS 17.0 statistical software package (SPSS, Inc., Chicago, IL, USA) for Windows. Normally distributed data are presented as the mean \pm standard error of the mean, whereas non-normally distributed data are presented as the median. Baseline comparisons between groups were performed using one-way analysis of variance for multiple comparisons, followed by Bonferroni correction. The effect of FA on NO production and biopterin and tHcy levels was assessed using Mann-Whitney U, Wilcoxon signed rank and t-tests for unpaired or paired data, as appropriate. A two-tailed value of $\mathrm{P}<0.05$ was considered to indicate statistical significance.

\section{Results}

Effect of FA on intracellular Hcy concentration. The concentration of Hcy in HUVECs treated with various doses of FA was investigated in normal and Hcy-stimulated cells. Cell lysates and culture medium were harvested after $24 \mathrm{~h}$ and were analyzed using HPLC to determine the cellular Hcy concentration. The levels of Hcy were found to be reduced in the high-dose FA groups in the cells not stimulated by Hcy; by contrast, the levels of Hcy in the Hcy-stimulated cells were increased $(\mathrm{P}<0.05$ Hcy group vs. high-dose FA groups). However, no significant differences were observed in the 0.1 and $1 \mathrm{nmol} / 1 \mathrm{FA}$ groups compared with the Hcy group, suggesting that the effect of FA on Hcy concentration is dose-dependent, with FA only capable of reducing Hcy concentration at high doses (Figs. 1 and 2).

Effect of FA on the intracellular level of BH4 and neopterin. To investigate whether FA could directly protect vascular endothelial cells, the effects of FA on BH4 and neopterin levels were investigated in Hcy-stimulated cells. BH4 is one of the most important cofactors of eNOS and is capable of reducing or reversing endothelial dysfunction by restoring the production of NO. HUVEC cultivation with FA for $24 \mathrm{~h}$ was observed to increase the level of BH4 (Fig. 3) and the BH4:neopterin ratio, particularly in the 5 and $10 \mathrm{nmol} / \mathrm{l} \mathrm{FA}$ treatment groups. However, FA was not found to reduce the level of intracellular neopterin (Fig. 4). Neopterin is a novel marker of inflammation in humans. Therefore, the data indicate that although FA appears to increase the activity of eNOS through increasing levels of the eNOS cofactor BH4, it is unable to reduce the level of inflammation in HUVECs.

Effect of FA on NO availability in endothelial cells. To further investigate the enhancement of eNOS coupling induced by FA, the intracellular concentration of NO was detected in Hcy-stimulated cells. FA was found to significantly increase NO levels, particularly in the 5 and $10 \mathrm{nmol} / \mathrm{l} \mathrm{FA}$ groups (37.86 \pm 12.34 and $38.451 \pm 11.23 \mathrm{nmol} / \mathrm{l})$ (Fig. 5), compared with the paired Hcy group $(26.21 \pm 9.24)(\mathrm{P}<0.001)$. These observations indicate that FA may enhance NO-mediated endothelial function through increasing e-NOS coupling (via 


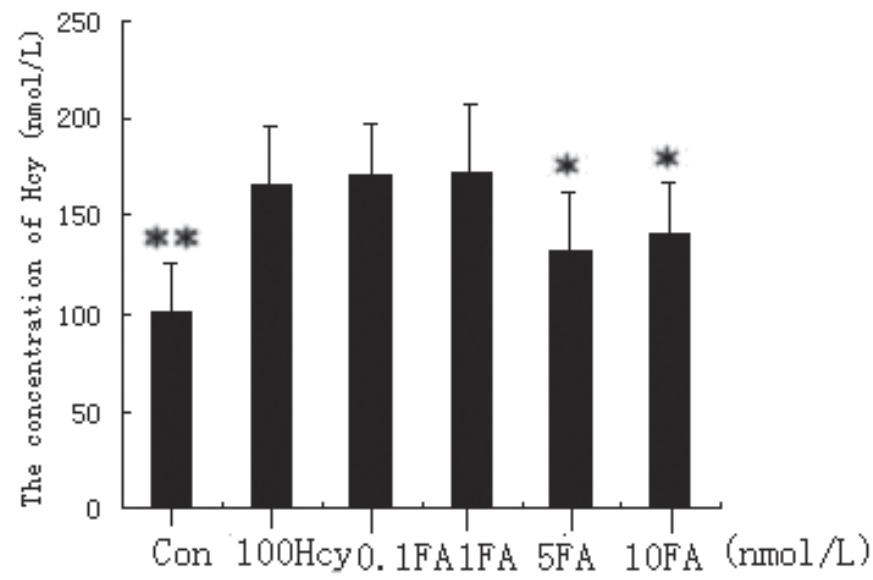

Figure 1. Effect of FA on Hcy concentration in human umbilical vein endothelial cells incubated with increasing concentrations of FA for $12 \mathrm{~h}$. Hcy levels remained unchanged in the control cells, but were significantly increased in cells following incubation with Hcy. Hcy production was reduced following incubation with FA, particularly in the high-dose FA groups. "P<0.05 and ${ }^{* *} \mathrm{P}<0.01$ vs. Hcy group. Hcy, homocysteine; FA, folic acid; Con, control.

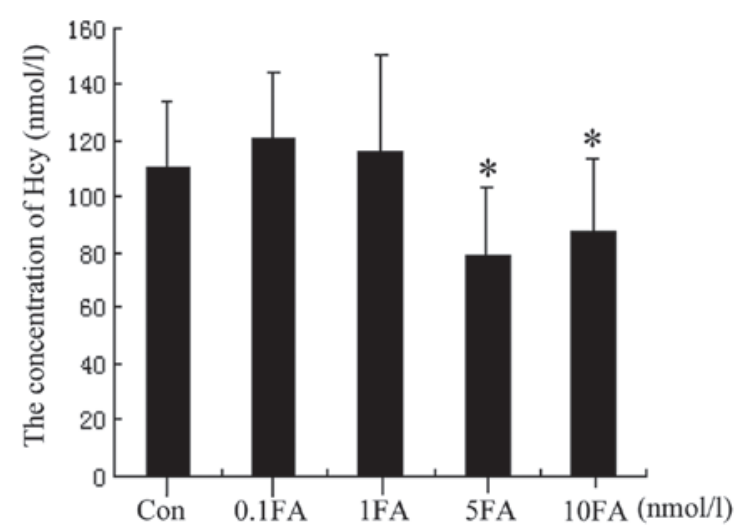

Figure 2. Effect of FA on Hcy concentration in human umbilical vein endothelial cells incubated with increasing concentrations of FA for $12 \mathrm{~h}$. Hcy concentrations remained unchanged in the low-dose FA groups compared with the control group, but were significantly decreased in the high-dose FA groups. "P<0.05 vs. control. Hcy, homocysteine; FA, folic acid; Con, control.

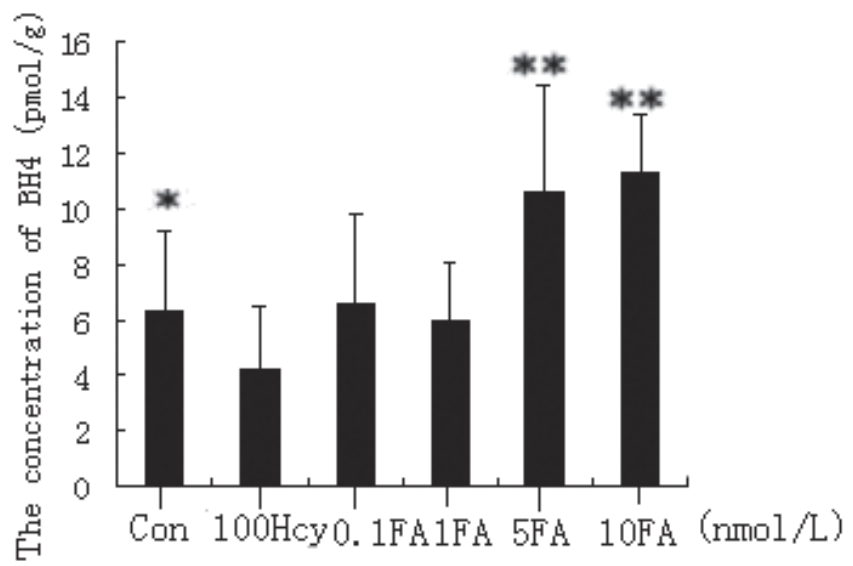

Figure 3. Effect of FA on BH4 levels in endothelial cells. Ex vivo incubation with FA for $12 \mathrm{~h}$ was found to increase BH4 levels compared with levels in the control and Hcy groups. No significant difference was observed in the low-dose FA groups compared with the control and Hcy. ${ }^{*} \mathrm{P}<0.05$ and ${ }^{* *} \mathrm{P}<0.01$ vs. Hcy group. Hcy, homocysteine; FA, folic acid; BH4, tetrahydrobiopterin; Con, control.

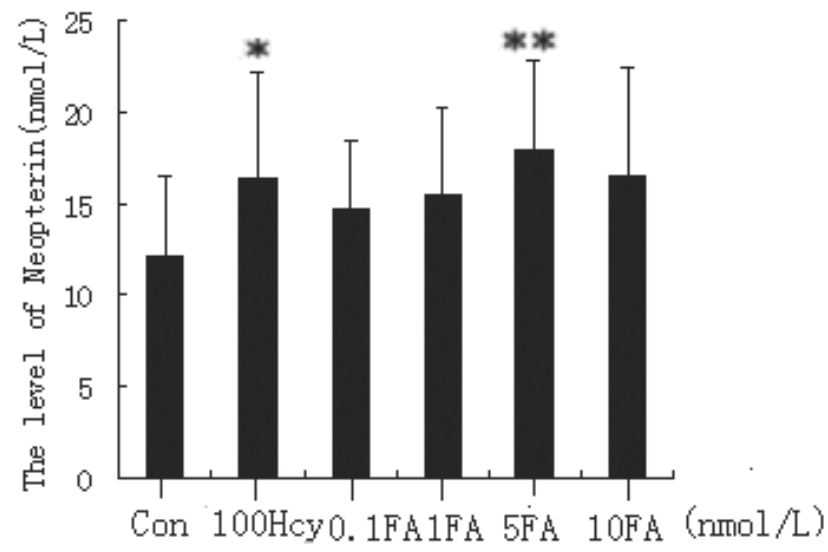

Figure 4. Neopterin scavenging by FA was assessed with Hcy. FA had no significant effect on neopterin scavenging. Treatment with various concentrations of FA for $12 \mathrm{~h}$ did not reduce levels of neopterin in endothelial cells. However, neopterin production was found to increase in cells incubated with Hcy. ${ }^{*} \mathrm{P}<0.05,{ }^{* *} \mathrm{P}<0.01$ vs con. Hcy, homocysteine; FA, folic acid; Con, control.

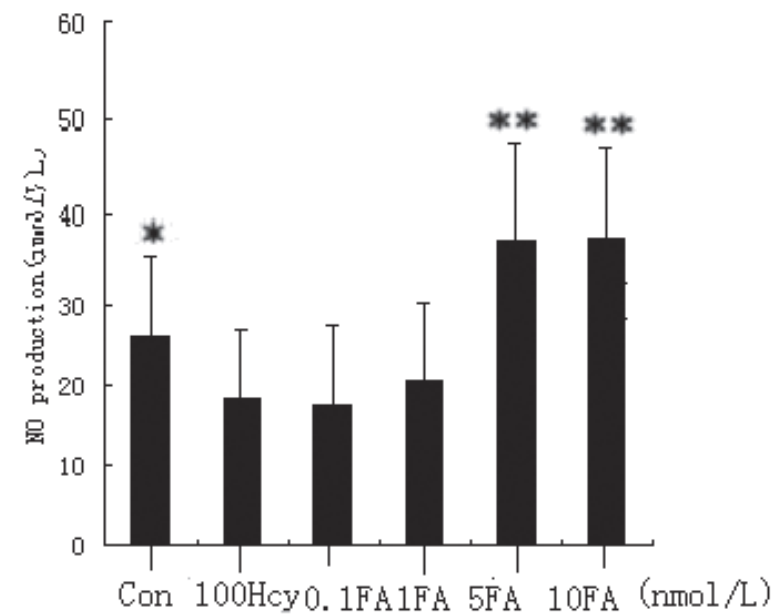

Figure 5. NO production was significantly increased following incubation with increasing concentrations of FA for $12 \mathrm{~h}$ compared with that in the control and Hcy groups. ${ }^{*} \mathrm{P}<0.05,{ }^{* *} \mathrm{P}<0.01$ vs. Hcy group. Hcy, homocysteine; NO, nitric oxide; FA, folic acid; Con, control.

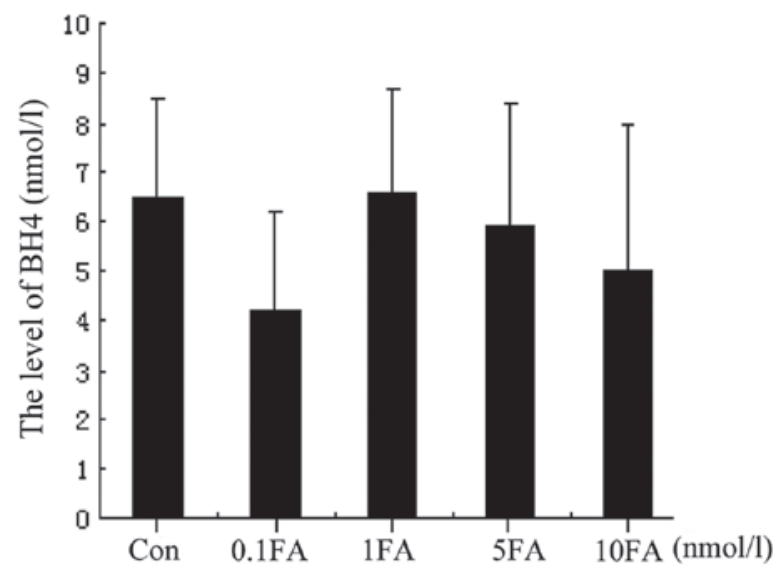

Figure 6. Cell BH4 levels in different FA treatment groups in normal endothelial cells. Following ex vivo incubation with increasing concentrations of FA for $12 \mathrm{~h}$, no significant differences were observed in the level of BH4 among the FA treatment and control groups. FA was not found to increase $\mathrm{BH} 4$ production in normal endothelial cells. Hcy, homocysteine; FA, folic acid; $\mathrm{BH} 4$, tetrahydrobiopterin; Con, control. 
increasing levels of the cofactor $\mathrm{BH} 4$ ) and $\mathrm{NO}$ production in endothelial cells.

The effect of FA on normal, unstimulated endothelial cells was also assessed. The results were inconsistent with those mentioned above. High doses of FA were found to reduce the levels of Hcy in normal endothelial cells; however, the levels of $\mathrm{BH} 4$ and $\mathrm{NO}$ production were not found to increase in the cells (Fig. 6).

\section{Discussion}

Numerous studies have reported that hyperhomocysteine causes vascular endothelial injury (9-11). Hyperhomocysteinemia is an independent risk factor for cerebral and cardiovascular diseases, due to its association with increased risks of thrombotic and atherosclerotic vascular diseases (12-15). Folate is a key cofactor for enzymes that metabolize Hcy, and has been used to reduce Hcy levels in humans (16). Although treatment with high doses of FA may be beneficial specifically in patients with high tHcy levels, benefits in other patients have been inconsistent (7). In the present study, high, but not low, FA doses were found to reduce the levels of Hcy and increase the concentrations of BH4 and NO, thus exhibiting a beneficial effect. However, FA was not found to protect normal endothelial cells through increases in $\mathrm{BH} 4$ and $\mathrm{NO}$.

Hcy is a non-protein-forming amino acid that is a product of $S$-adenosyl methionine-dependent transmethylation reactions following the action of $S$-adenosyl-L-homocysteine hydrolase. In order to prevent Hcy accumulation and the development of hyperhomocysteinemia, Hcy is either remethylated back to methionine or irreversibly catabolized through the trans-sulfuration pathway to cysteine. It is well established that hyperhomocysteinemia is an independent risk factor for atherosclerosis, since it can promote LDL oxidation and internalization by macrophages, leading to the oxidation of numerous substances in the body, including BH4, eNOS and NO. Hcy is capable of directly accelerating NO degradation. Endothelial dysfunction is a major marker of cardiovascular risk and is characterized by a reduced production/availability of NO and/or an imbalance between endothelium-derived relaxation and contracting factors and oxidants $(9,17)$. In the present study, FA was found to significantly reduce the levels of Hcy in normal cells and in cells treated with Hcy.

Neopterin, a by-product of the GTP pathway, is produced by activated macrophages upon stimulation with interferon- $\gamma$ released from $\mathrm{T}$ lymphocytes, and is an activation marker for monocytes/macrophages (18-19). Inflammatory processes have an important role in the pathogenesis of atherosclerotic plaque and its complications, and monocyte/macrophage activation contributes to the processes associated with atherosclerosis and plaque vulnerability. Increased levels of inflammatory biomarkers have an important role in the initiation and progression of atherosclerotic vascular disease (20). Therefore, neopterin is a key factor in numerous diseases. However, in the present study, FA was not observed to reduce the concentration of neopterin in endothelial cells, suggesting that FA treatment did not change the inflammatory reaction or reduce the development of atherosclerosis. Inflammation is an important factor in the development of atherosclerosis. Without improving the inflammation status in the body, the diseases are likely to continue. This may explain why FA does not have long-term effects for cardiovascular diseases and may restrict its application in the clinic.

The role of BH4 in the control of NOS activity was initially identified almost 20 years ago. It is now well established that $\mathrm{BH} 4$ is a key factor required for the enzymatic activity of all three of the NOS isoforms; however, eNOS, which is important for endothelial cells, has attracted the most attention. Numerous studies have demonstrated that the chemical inactivation of $\mathrm{BH} 4$ by oxidative stress causes uncoupling of eNOS, which catalyzes the formation of the superoxide anion and oxidizes $\mathrm{NO}$ to $\mathrm{NO}_{2}^{-}$. By contrast, if $\mathrm{BH} 4$ levels increase, eNOS becomes coupled, which catalyzes the formation of NO (21-22). In summary, NO bioavailability is a result of NO production and degradation. NO production is catalyzed by eNOS, which requires $\mathrm{BH} 4$ as an essential cofactor. In the present study, $\mathrm{BH} 4$ production was investigated in normal and stimulated endothelial cells. High doses of FA were found to increase the levels of $\mathrm{BH} 4$ in the stimulated cells; however, the concentration of $\mathrm{BH} 4$ remained unchanged in the normal cells. Therefore, high-dose FA treatment may be beneficial in patients with high tHcy levels, but benefits for other individuals are uncertain.

Folate is involved in numerous reactions in the body and folate deficiency has been implicated in various diseases, including atherosclerosis, neural tube defects and cancer. Folate is used widely in the clinic, for example in neuropsychiatric and neurological disorders, anemia and cardiovascular diseases. An increasing number of studies have focused the application of folate in cardiovascular diseases. However, the conclusions are not consistent. Therefore, the potential for the application of folate in cardiovascular diseases is yet to be elucidated. In the present study, high doses of FA were found to protect endothelial cells by increasing the levels of $\mathrm{BH} 4$ and the production of NO. However, FA was not observed to reduce inflammation, which may explain why folate is not capable of providing long-term effects for cardiovascular diseases. Similar results, with regard to BH4 and NO, were not found in unstimulated endothelial cells.

In conclusion, in the present study, high doses of FA were observed to be important for injured endothelial cells, but not for normal, unstimulated endothelial cells. Although FA was found to have a beneficial effect on injured cells, the underlying mechanism is yet to be elucidated. Furthermore, the reason why FA was not found to be beneficial for normal cells remains unclear. Further investigations are required to elucidate these mechanisms and provide evidence for the use of folate in the clinic.

\section{References}

1. Hamon CG, Blair JA and Barford PA: The effect of tetrahydrofolate on tetrahydrobiopterin metabolism. J Ment Defic Res 30: 179-183, 1986.

2. Moens AL and Kass DA: Tetrahydrobiopterin and cardiovascular disease. Arterioscler Thromb Vasc Biol 26: 2439-2444, 2006.

3. Coppola A, Davi G, De Stefano V, et al: Homocysteine, coagulation, platelet function, and thrombosis. Semin Thromb Hemost 26: 243-254, 2000. 
4. Bunout D, Garrido A, Suazo M, et al: Effects of supplementation with folic acid and antioxidant vitamins on homocysteine levels and LDL oxidation in coronary patients. Nutrition 16: 107-110, 2000.

5. Voutilainen S, Morrow JD, Roberts LJ II, et al: Enhanced in vivo lipid peroxidation at elevated plasma total homocysteine levels. Arterioscler Thromb Vasc Biol 19: 1263-1266, 1999.

6. Woo KS, Chook P, Lolin YI, et al: Folic acid improves arterial endothelial function in adults with hyperhomocystinemia. J Am Coll Cardiol 34: 2002-2006, 1999.

7. Antoniades C, Shirodaria C, Warrick N, et al: 5-methyltetrahydrofolate rapidly improves endothelial function and decreases superoxide production in human vessels: effects on vascular tetrahydrobiopterin availability and endothelial nitric oxide synthase coupling. Circulation 114: 1193-1201, 2006.

8. Alp NJ, Mussa S, Khoo J, et al: Tetrahydrobiopterin-dependent preservation of nitric oxide-mediated endothelial function in diabetes by targeted transgenic GTP-cyclohydrolase I overexpression. J Clin Invest 112: 725-735, 2003.

9. de Andrade CR, Leite PF, Montezano AC, et al: Increased endothelin-1 reactivity and endothelial dysfunction in carotid arteries from rats with hyperhomocysteinemia. Br J Pharmacol 157: 568-580, 2009.

10. Mahadeo KM, Dhall G, Panigrahy A, et al: Subacute methotrexate neurotoxicity and cerebral venous sinus thrombosis in a 12-year-old with acute lymphoblastic leukemia and methylenetetrahydrofolate reductase (MTHFR) C677T polymorphism: homocysteine-mediated methotrexate neurotoxicity via direct endothelial injury. Pediatr Hematol Oncol 27: 46-52, 2010.

11. Papatheodorou L and Weiss N: Vascular oxidant stress and inflammation in hyperhomocysteinemia. Antioxid Redox Signal 9: 1941-1958, 2007.

12. Vizzardi E, Bonadei I, Zanini G, et al: Homocysteine and heart failure: an overview. Recent Pat Cardiovasc Drug Discov 4: 15-21, 2009.
13. Guilland JC, Favier A, Potier de Courcy G, et al: Hyperhomocysteinemia: an independent risk factor or a simple marker of vascular disease? 1. Basic data. Pathol Biol (Paris) 51: 101-110, 2003 (In French).

14. Cattaneo M: Hyperhomocysteinemia and thrombosis. Lipids 36 (Suppl): S13-S26, 2001.

15. Herrmann W: The importance of hyperhomocysteinemia as a risk factor for diseases: an overview. Clin Chem Lab Med 39: 666-674, 2001.

16. Krebs HA, Hems R and Tyler B: The regulation of folate and methionine metabolism. Biochem J. 158: 341-353, 1976.

17. Zhang JG, Wang LZ, Han XQ, et al: The pathogenic mechanism of homocysteine -induced endothelial nitric oxide synthase dysfunction and the antagonistic effects by folic acid. Fen Zi Xi Bao Sheng Wu Xue Bao 40: 17-23, 2007 (In Chinese).

18. Aisaki K, Tsuboi I, Harada T, et al: Neopterin, inflammation-associated product, prolongs erythropoiesis suppression in aged SAMP1 mice due to senescent stromal-cell impairment. Exp Biol Med (Maywood) 237: 279-286, 2012.

19. Yadav AK, Sharma V and Jha V: Association between serum neopterin and inflammatory activation in chronic kidney disease. Mediators Inflamm 2012: 476979, 2012.

20. Zamani P, Ganz P, Libby P, et al: Relationship of antihypertensive treatment to plasma markers of vascular inflammation and remodeling in the Comparison of Amlodipine versus Enalapril to Limit Occurrences of Thrombosis study. Am Heart J 163: 735-740, 2012

21. Hyndman ME, Verma S, Rosenfeld RJ, et al: Interaction of 5-methyltetrahydrofolate and tetrahydrobiopterin on endothelial function. Am J Physiol Heart Circ Physiol 282: H2167-H2172, 2002.

22. Ronco AM, Llanos M, Tamayo D and Hirsch S: 5-methyltetrahydrofolic acid stimulates endothelin-1 production in low density lipoprotein-treated human endothelial cells. Nutr Metab Cardiovasc Dis 17: 188-194, 2007. 